\title{
Addressing the Unmet Needs of Measuring Vascular Ageing in Clinical Practice-European COoperation in Science and Technology Action VascAgeNet
}

\author{
Rachel Emma Climie ${ }^{1,2,3}$, Christopher Clemens Mayer ${ }^{4, *}$, Rosa Maria Bruno ${ }^{1,5}$, Bernhard Hametner $^{4}$ \\ ${ }^{I}$ INSERM U970 Paris-Centre de Recherche Cardiovasculaire (PARCC), Paris, France \\ ${ }^{2}$ Sports Cardiology and Diabetes and Population Health, Baker Heart and Diabetes Institute, Melbourne, Australia \\ ${ }^{3}$ Blood Pressure Research Group, Menzies Institute for Medical Research, College of Health and Medicine, University of Tasmania, Australia \\ ${ }^{4}$ Center for Health \& Bioresources, Biomedical Systems, AIT Austrian Institute of Technology GmbH, Giefinggasse 4, Vienna 1210, Austria \\ ${ }^{5}$ Department of Pharmacology, Université Paris Descartes - Université de Paris, Paris, France
}

\section{ARTICLE INFO}

Article History

Received 29 November 2019

Accepted 21 March 2020

\section{Keywords}

COST Action

VascAgeNet

network

vascular ageing

study protocol

\begin{abstract}
Cardiovascular Disease (CVD) is the leading cause of morbidity and mortality worldwide, regardless of gender, ethnicity or income. Evidence that vascular age, as opposed to chronological age, is better related to the prognosis of CVD is convincing. Despite recent technological advances for the measurement of vascular ageing, unmet needs remain which currently limit the assessment of vascular ageing in routine clinical practice. These unmet needs include: (1) lack of easy-to-use, affordable techniques/devices, (2) insufficient validation in clinical settings, (3) fragmentation of expertise in few research laboratories globally, and (4) lack of investigator/research driven intervention studies and head-to-head comparisons between different measurement techniques. This paper summarises how a recently awarded European COoperation in Science and Technology (COST) Action on Vascular Ageing (VascAgeNet) will address these unmet needs. The broad aim of the COST Action VascAgeNet is to establish a network which will work to refine, harmonize and promote the use of vascular ageing biomarkers, in order to improve clinical practice and to reduce the burden of CVD globally.
\end{abstract}

(C) 2020 Association for Research into Arterial Structure and Physiology. Publishing services by Atlantis Press International B.V. This is an open access article distributed under the CC BY-NC 4.0 license (http://creativecommons.org/licenses/by-nc/4.0/).

\section{INTRODUCTION}

Vascular ageing, the decline in vascular structure and function, is an integrated marker of overall cardiovascular risk burden on the vasculature over time and ultimately leads to end organ damage in the heart, brain and kidney [1]. While age-dependent arterial damage typically appears in the fifth or sixth decade of life, there is wide variability between individuals with some displaying early vascular ageing. Exposure to a number of environmental and genetic factors as early as during childhood or even during fetal life promotes the development and accumulation of subclinical vascular changes that directs an individual toward a trajectory of early vascular ageing [2]. This has led to the concept that vascular age, as opposed to chronological age, may be better related to the prognosis of Cardiovascular Disease (CVD) [3,4].

While recent research and technological advances relating to vascular ageing have been promising, there are still a number of unmet needs that remain and limit the measurement of vascular ageing in routine clinical practice. These unmet needs include:

(1) Lack of easy-to-use, affordable techniques/devices that can be used in large numbers of subjects including low-income countries;

"Corresponding author. Email: christopher.mayer@ait.ac.at

Peer review under responsibility of the Association for Research into Arterial Structure and Physiology
(2) Insufficient validation in clinical settings. While a large body of evidence on vascular biomarkers has been accumulated in the last 30 years, no biomarker fulfils all of the criteria to be considered a surrogate endpoint;

(3) Fragmentation of expertise in few research laboratories globally and lack of uptake of new concepts within the wider clinical audience;

(4) Lack of investigator/researcher driven intervention studies, especially concerning the impact of pharmacological treatment on vascular biomarkers and head-to-head comparisons between different measurement techniques.

This paper summarises how a recently awarded European COoperation in Science and Technology (COST) Action on vascular ageing [CA18216 - Network for Research in Vascular Ageing, VascAgeNet, www.vascagenet.eu, Action duration 4 years (November 5 2019-November 4 2023)] will address these unmet needs. Below, the objectives of the COST Action VascAgeNet and how these will be addressed over the next 4 years is outlined and the expected impact of the Cost Action is described.

\section{THE PURPOSE OF COST ACTIONS}

COoperation in Science and Technology is a funding organisation for research. COST Actions support research initiatives 
across Europe by enabling scientists to share and discuss their research with their peers, rather than funding research per se. The purpose of COST Actions is to create European research networks to facilitate collaborative, international research (https:// www.cost.eu/who-we-are/about-cost/). COST Actions focus on (1) openness (i.e., partners can join at any time); (2) inclusiveness with respect to less connected research communities, Early Career Investigators (ECIs) and equal gender representation; (3) interdisciplinary (i.e., bringing basic research, engineering and industry together) and; (4) intersectional (i.e., combining universities, hospitals, research organization, small and medium-sized enterprises etc.).

\section{OBJECTIVES OF COST ACTION VascAgeNet}

The broad aim of COST Action VascAgeNet is to establish a network which will work to refine, harmonize and promote the use of vascular ageing biomarkers, in order to improve clinical practice and to reduce the burden of CVD globally.

The main objectives of the VascAgeNet are to:

(1) Coordinate new European research on vascular ageing at an international level;

(2) Coordinate ongoing research in an interdisciplinary (medical, engineering, business), inter-country (the COST Action already involves more than 150 representatives from more than 30 countries and two international partners USA and Australia, at the time of submission) and intersectoral (academic, research, business) network;

(3) Connect and promote knowledge transfer between researchers, practitioners, patients and companies.

Specifically, the multidisciplinary network will work to:

\subsection{Refine}

- Identify and refine new technologies for the assessment of vascular ageing;

- Achieve better understanding of the underlying mechanisms of vascular ageing;

- Provide new evidence about the pathophysiological mechanisms leading to the development of vascular ageing to be used for the study on new therapeutic strategies;

- Determine in whom the measurement of vascular ageing is likely to be of most benefit and the cost effectiveness of this approach.

\subsection{Harmonise}

- Design and implement a data registry to enable multi-centre research;
- Define innovative big data approaches for the identification of vascular ageing risk factors to be used for the implementation of prevention and screening programs;

- Initiate peer network driven intervention studies on vascular ageing;

- Facilitate the development of new techniques/devices via validation (e.g., head-to-head comparison);

- Establish priorities for future translational research via the identification and promotion of basic and pre-clinical experimental protocols.

\subsection{Promote}

- Advocate for the inclusion of vascular ageing in international medical guidelines;

- Encourage measurement of vascular ageing in primary practice through education and dissemination of information to general practitioners;

- Promote vascular ageing prevention with a particular focus on end-users (patients), those who interact with them (e.g., specialists working in medical practices, general practitioners, nurses, pharmacists, patient-organisations, journalists), low-income countries and various health care systems.

\section{CAPACITY-BUILDING OBJECTIVES}

The capacity-building objectives of VascAgeNet will help to fulfil the above objectives. In particular, they will ensure the promotion of a vascular ageing culture, spreading the technological advances obtained to all countries and the inclusion international partners (USA and Australia) will ensure this happens on a global level. To improve the clinical impact of the vascular ageing concept, the network will foster strategies aimed at scientific societies, governments, health care systems and the research community. Thus, the COST Action will:

\subsection{Refine}

- Develop an interdisciplinary collaborative network covering excellence in science and technology across Europe to foster joint research concerning arterial structure, function and physiology to enhance and exchange knowledge and expertise.

- Combine different research fields with start-ups/industry to create a multidisciplinary and translational approach for the achievement of important breakthroughs.

\subsection{Harmonise}

- Offer interdisciplinary opportunities for co-operation between scientists and physicians/clinicians.

- Bring basic research, applied research and industry together, to translate research from bench to bedside. 


\subsection{Promote}

- Attract the next generation of ECIs to research in arterial structure and physiology and support their interdisciplinary education through training schools, workshops and conferences.

- Disseminate knowledge and experience resulting from this network by publications (reports and papers), workshops, seminars, a final conference, public awareness days, an easily accessible user-friendly website and social media.

- Proactively involve specific target groups including ECIs, participants from Inclusiveness Target Countries (ITCs) and equal gender balance.

- Enlarge the network by involving external experts in network activities.

\section{DESCRIPTION OF WORKING GROUPS' OBJECTIVES}

Five Working Groups (WG) will work in collaboration and address specific objectives related directly to the main objectives of the COST Action VascAgeNet. The objectives of each working group are described below and their interaction depicted in Figure 1. While WGs 2-4 will be content-driven, the exchange and collaboration between all WGs will be ensured by WG1, and WG5 will be responsible for the dissemination of information. Members of the network from both European and international partner countries are invited to join the WGs.

\subsection{WG1: Dynamic Exchange}

This capacity building WG will coordinate activities between WGs, to ensure a fruitful interaction between different professionals who might tend to concentrate in the same WG (e.g. basic scientists in WG2, industry representative in WG3). Furthermore, WG1 will work actively to enlarge the network, to fulfil the principle of openness inspiring COST actions. Finally, given that the COST funding is available for the next 4 years, exploration and application for future EU funding beyond this to support research stemming from the network will be another crucial task for WG1. The list of objectives for all WGs, as in the Memorandum of Understanding (https://www.cost.eu/actions/CA18216/) are detailed in Table 1.

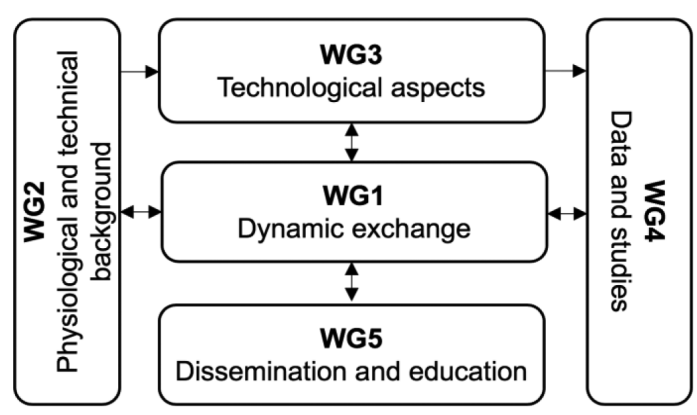

Figure 1 The COST Action VascAgeNet working groups (WG).
Table 1 The main objectives of the VascAgeNet working groups

\begin{tabular}{|c|c|}
\hline$T$ & $\begin{array}{l}\text { Ensure the interdisciplinary collaboration within } \\
\text { the network to enhance and exchange knowledge } \\
\text { and expertise } \\
\text { - Enlarge the network during the course of the } \\
\text { COST Action and to build collaborations with } \\
\text { external experts } \\
\text { - Proactively involve ECIs, ITCs, under-represented } \\
\text { genders } \\
\text { - Create a multidisciplinary and translational } \\
\text { approach for the achievement of important break- } \\
\text { throughs especially by linking research and industry } \\
\text { - Explore and promote subsequent funding possibil- } \\
\text { ities based on the COST Action's content }\end{array}$ \\
\hline g Group 2 & $\begin{array}{l}\text { - Refine the understanding of the underlying mech- } \\
\text { anisms of vascular ageing } \\
\text { - Reach consensus on the application of different math- } \\
\text { ematical models used for vascular ageing assessment } \\
\text { - Identify future targets for treatment of early } \\
\text { vascular ageing in a translational approach }\end{array}$ \\
\hline Working Group 3 & $\begin{array}{l}\text { - Standardise, refine and harmonise existing and } \\
\text { new technological approaches for the assessment } \\
\text { of vascular ageing } \\
\text { - Perform a head-to-head comparison of new tech- } \\
\text { niques and devices }\end{array}$ \\
\hline Wo & $\begin{array}{l}\text { - Create a big data registry for harmonizing available } \\
\text { data and studies for enabling multicenter research } \\
\text { initiatives } \\
\text { - Identify new risk factors for vascular ageing by } \\
\text { means of big data approaches } \\
\text { - Promote interventional studies }\end{array}$ \\
\hline Working Group 5 & $\begin{array}{l}\text { - Promote a vascular ageing culture and the use of } \\
\text { technologies and preventative strategies } \\
\text { - Disseminate results addressing various stake- } \\
\text { holders, e.g., scientists, doctors, nurses, patients, } \\
\text { patient associations, representatives from health } \\
\text { care systems, guideline committees } \\
\text { - Educate clinicians, ensuring involvement from } \\
\text { ECIs, ITCs and a gender balance }\end{array}$ \\
\hline
\end{tabular}

ECIs, Early Career Investigators; ITCs, Inclusiveness Target Countries.

\subsection{WG2: Physiological and Technical Background - From Bench to Bedside}

The activities of this content-driven WG will be to summarise current knowledge on mechanisms of vascular ageing derived from basic research, and to establish research priorities for integration of novel pathways into the framework of integrative physiology and translation into clinical research and drug development. Furthermore, this WG will have the task to reach consensus on the application, differences, interplay, limitations and potential complementarity of different mathematical models and techniques used for vascular ageing assessment, thus advancing their potential clinical application.

\subsection{WG3: Technological Aspects - Supporting Technology Breakthroughs}

The main activity of this group will be to promote standardization and clarity in the field of development of devices for the assessment 
of vascular ageing, to overcome the existing fragmentation of knowledge and approaches. Accordingly, this WG will work on an updated consensus document to be used in clinical practice and for the validation of new techniques. Furthermore, WG3 will create a framework to support the development and validation and use of innovative techniques, not only by supporting head-tohead comparisons between techniques, but also by ensuring the dialogue between healthcare professionals and industry. In particular, a large-scale survey among clinicians, aimed at identifying gaps for application of innovative techniques into routine clinical practice, will be launched. Finally, a cost-effectiveness analysis of patient management strategies based on vascular aging biomarkers will be performed.

\subsection{WG4: Data and Research Studies - Big Data Approaches}

Working group 4 will work to design and implement a concept for a large and comprehensive data registry, identifying appropriate strategies for data collection and management, as well as methods for ensuring high data quality. The data registry, including available datasets in which vascular aging biomarkers were used, will help consolidate existing evidence on the usefulness of vascular aging biomarkers for cardiovascular risk prediction and for tracking efficacy of treatments, and will help to identify novel risk factors for vascular ageing. A focus of this group will be on promoting implementation of big data approaches. Furthermore, the registry will constitute the basis for facilitating future shared initiatives, including the design of a novel generation of interventional trials. A number of drug treatments already available in clinical practice have demonstrated potential for slowing or regressing vascular aging, as recently reviewed [1,4]. The constitution of the European registry will make it possible to run registry-based, pragmatic interventional trials, a powerful tool for conducting studies efficiently and cost-effectively [5].

\subsection{WG5: Dissemination and Education - Promoting a Vascular Ageing Culture}

This capacity-building WG will coordinate the dissemination of the results of the content-driven WGs, directed toward a wide audience including healthcare professionals, the scientific community and general population, identifying the most appropriate communication tool for each target population. Furthermore, it will support the organization of the educational activities of the COST Action, mostly targeted on ITCs and ECIs. This will include the organization of technical workshops, an annual training school as well as sessions at international meetings.

Details regarding the leadership structure including the WG leaders of VascAgeNet can be found in the Online Supplement or at http://vascagenet.eu/team (status: March 5 2020). Country representatives can be found at https://www.cost.eu/actions/CA18216/.

\section{IMPACT}

The expected impact of the Action is summarized below.

\subsection{Short-term Impact}

- The development of an interdisciplinary network consisting of members from research and industry that will drive scientific and technological advances and generate breakthroughs.

- The identification of gaps in the current scientific knowledge regarding the measurement of vascular ageing which will allow for scientific developments over the longer-term to address these gaps.

- Increase in knowledge of Action participants on vascular ageing leading to the inclusion of new knowledge to teaching and training activities, curricula and continuous education in medicine.

\subsection{Long-term Impact}

- Through enhanced cooperation between research and industry, the increased cross-talk between researchers/clinicians trialling new technologies will lead to new technologies and enhance translation of knowledge from bench to bedside. This will also aid scientific and technological breakthroughs.

- Building a data registry and fostering big data approaches will allow for reliable risk prediction models to be created, to identify those at increased risk due to early vascular ageing. This will have significant societal impact in terms of reducing the burden of vascular ageing and thereby reducing health care costs. This will also allow for head-to-head comparisons between different techniques measuring vascular ageing and to identify the most reliable techniques which can be applied in routine clinical practice.

- It is currently unknown which biomarker responds most effectively to pharmacological and non-pharmacological induced improvements in vascular health. Therefore, by planning a peer network-driven intervention study to assess the effect of different therapies on vascular ageing, the Action will have long-term impact in terms of the design of targeted therapies.

- By harmonising and refining techniques for vascular ageing assessment, currently available techniques will be directly comparable in terms of effectiveness, simplicity/complexity of use, accuracy and costs, which will allow for differentiation of applicability at different stages of prevention, diagnosis and therapy.

- By promoting concepts relevant to vascular ageing in low-income countries, this Action will help to reduce the risk related to vascular ageing and (premature) CVD over the long-term, and will have significant societal impact on population health as well as the healthcare systems in low-income countries.

\section{CONCLUSION}

Over the next 4 years, the VascAgeNet interdisciplinary network will work together to address the unmet needs impeding the widespread use of vascular aging biomarkers in the clinical practice. All interested parties are invited to join VascAgeNet, to advance research and technological developments related to vascular ageing, enhance clinical care and, ultimately, improve the quality of life for many people worldwide. 


\section{CONFLICTS OF INTEREST}

The authors declare they have no conflicts of interest.

\section{AUTHORS' CONTRIBUTION}

Each author contributed important intellectual content during manuscript drafting or revision. REC, CCM, RMB and BH funding acquisition; CCM project chair; REC, CCM, RMB and $\mathrm{BH}$ writing (original draft) the manuscript.

\section{FUNDING}

COST Action CA18216 is supported by COST (European Cooperation in Science and Technology, www.cost.eu). COST is a funding agency for research and innovation networks. Their Actions help connect research initiatives across Europe and enable scientists to grow their ideas by sharing them with their peers. This boosts their research, career and innovation.

\section{REFERENCES}

[1] Climie RE, van Sloten TT, Bruno RM, Taddei S, Empana JP, Stehouwer CDA, et al. Macrovasculature and microvasculature at the crossroads between type 2 diabetes mellitus and hypertension. Hypertension 2019;73:1138-49.

[2] Olsen MH, Angell SY, Asma S, Boutouyrie P, Burger D, Chirinos JA, et al. A call to action and a lifecourse strategy to address the global burden of raised blood pressure on current and future generations: the Lancet Commission on hypertension. Lancet 2016;388:2665-712.

[3] Nilsson PM, Boutouyrie P, Laurent S. Vascular aging: a tale of EVA and ADAM in cardiovascular risk assessment and prevention. Hypertension 2009;54:3-10.

[4] Hamczyk MR, Nevado RM, Barettino A, Fuster V, Andrés V. Biological versus chronological aging: JACC focus seminar. J Am Coll Cardiol 2020;75:919-30.

[5] James S, Rao SV, Granger CB. Registry-based randomized clinical trials-a new clinical trial paradigm. Nat Rev Cardiol 2015;12:312-6. 